\title{
Evaluación de las Políticas Nacionales de Información Geoespacial de Ecuador vinculadas con la implementación de IDE institucionales
}

\author{
Rocío Narváez-Benalcázar* \\ Fernanda León-Pazmiño* \\ Miguel-Ángel Bernabé-Poveda* \\ María Laura Rubio**
}

Recibido el 28 de agosto; aceptado el 26 de septiembre de 2016

\begin{abstract}
The management of the country's territory is closely related to the Geographical Information (GI) available and its accessibility. These would be the fundamental principles of a Spatial Data Infrastructure (SDI) supported by a consolidated legal framework. In Ecuador, the National Geospatial Information Policy, established in 2010 by the National Council of Geoinformatics (CONAGE) is the legal framework that all institutions must comply with in the generation, update, use, distribution, delivery, exchange and sale of the country's geoinformation. With this background, two questions arise: (a) What has been the degree of implementation of that legislation in state institutions? and (b) How has it influenced the implementation of institutional SDI? This article aims to respond to these concerns through a methodology of qualitative and quantitative analysis, established from the results of a diagnosis survey. The survey was applied to 20 public institutions responsible for the official GI generation. The results show the degree of compliance with those policies and major problems or deficiencies found in the application process over the last six years (2010-2016).
\end{abstract}

* Instituto Geográfico Militar (IGM), Seniergues E4-676 y Gral. Telmo Paz y Miño, El Dorado, Quito, Ecuador, correos electrónicos: rocio.narvaez@mail.igm.gob.ec; fernanda.leon@mail.igm.gob.ec; ma.bernabe@gmail.com

** Universidad Nacional del Sur (UNS), Avenida Alem 1253, Bahía Blanca, Argentina, correo electrónico:mrubio@uns.edu.ar 
Key words: Politics, geospatial information, geoinformation, access, use, government institutions, Ecuador.

\section{Resumo}

Atualmente, a gestão territorial de um país está interligada com a Informação Geográfica (IG). A disponibilização e a acessibilidade destas informações são princípios fundamentais de uma Infraestrutura de Dados Espaciais, amparados por um marco legal consolidado. No Equador, essa base jurídica é instituída pelas Políticas Nacionais de Informação Geoespaciais, estabelecidas em 2010 pelo Conselho Nacional de Geoinformática (CONAGE). Todas as instituições do país devem considerar para a geração, atualização, utilização, compartilhamento, distribuição, transferência e venda de geoinformação, os critérios constituídos por tais políticas. Diante do exposto, surgem duas questões: Qual tem sido o grau de implementação dessa legislação nas instituições do Estado? Como tem influenciado a implementação das IDE institucionais? No presente artigo, procura-se responder estas questões através de uma pesquisa que foi aplicada a 20 instituições públicas responsáveis pela geração oficial da IG. Os resultados obtidos indicaram o grau de cumprimento destas políticas e os principais problemas ou dificuldades encontradas nos processos de sua aplicação nos últimos seis anos (2010-2016).

Palavras chave: Políticas, informação geoespacial, geoinformação, acesso, uso, instituições do Estado.

\section{Resumen}

En la actualidad, la gestión del territorio de un país, se encuentra estrechamente relacionada con la Información Geográfica (IG) disponible y con su accesibilidad. Estos serían los principios fundamentales de una Infraestructura de Datos Espaciales (IDE) amparada en un marco legal consolidado que la respalde. En Ecuador, este marco legal son las Políticas Nacionales de Información Geoespacial, establecidas en el año 2010 por el Consejo Nacional de Geoinformática (CONAGE), que todas las instituciones deben acatar en la generación, actualización, uso, difusión, entrega, intercambio y venta de la geoinformación del país. Con este antecedente, se plantean dos interrogantes, ¿cuál ha sido el grado de implementación de dicha normativa en las instituciones del Estado? y ¿cómo ha influido en la implementación de las IDE institucionales? Este artículo pretende dar respuesta a estas inquietudes mediante una metodología de análisis cualicuantitativo, establecida a partir de los resultados de una encuesta de diagnóstico. La misma fue aplicada a 20 instituciones públicas responsables de la generación oficial de IG. Los resultados obtenidos, muestran el grado de cumplimiento de 
dichas políticas y los principales problemas o deficiencias encontrados en el proceso de su aplicación en los últimos seis años (2010-2016).

Palabras clave: Políticas Nacionales de Información Geoespacial, geoinformación, acceso, usos, instituciones gubernamentales, Ecuador.

\section{Introducción}

Los datos geográficos son nombres, características y localizaciones de entidades geográficas de la Tierra. Sirven para generar multitud de productos tales como mapas topográficos, mapas temáticos, simuladores de eventos naturales, mapas en tiempo real y muchos más. Disponer de datos geográficos y transformarlos en información geográfica (IG) mediante la combinación de ellos es una manera de acercarse a la realidad de un territorio para comprenderlo mejor. Si además, se organiza en un sistema jerárquico y dinámico, que permita no sólo disponer de ella sino que también proporcione herramientas para la gestión y análisis por parte de los usuarios, posibilita mejorar las decisiones que se tomen sobre ese territorio (IGN, 2012). Ese es el principio en el que se basan las Infraestructuras de Datos Espaciales (IDE) que se convierten en el instrumento clave para localizar, acceder y usar la IG almacenada en diferentes fuentes a través de Internet.

Para que este uso extensivo de la IG ocurra en todos los niveles debe existir un compromiso por parte de las instituciones generadoras (o un mandato oficial) que la ponga a disposición de los usuarios, con las restricciones de uso requeridas. Debe además garantizar el fácil acceso y la interoperabilidad de la información proveniente de cualquier institución, resumido en un componente político de la IDE (Bernabé y López, 2012).

En este marco, el presente trabajo tiene como objetivo evaluar la existencia y disponibilidad de la información oficial y su grado de conformidad con las disposiciones oficiales de Ecuador.

\section{Antecedentes}

\section{Consideraciones políticas de la IDE de Ecuador}

La Constitución de la República de Ecuador, establece en su artículo 275 la responsabilidad del Estado de planificar el desarrollo del país, de forma articulada, equitativa, participativa y descentralizada. Para ello, se han creado en Ecuador, desde el año 2008, diversos marcos jurídicos e institucionales que permiten concertar la generación y ejecución de un Plan Nacional de Desarrollo.

Un elemento fundamental en esta planificación es el territorio, identificado como "el espacio de concreción de la política pública" (SENPLADES, 2009, p. 12). Ese espacio lo visualizan los actores a través de la información geográfica, 
compuesta por varios conjuntos de datos específicos, que debe ser generado por distintas instituciones gubernamentales.

El Consejo Nacional de Geoinformática (CONAGE), creado en 2004, dependiente de la Presidencia de la República, tiene, entre otras responsabilidades, la de:

...proponer la normativa necesaria para reglamentar la generación, almacenamiento, distribución, comercialización, actualización, uso, derechos de autor y otros aspectos relativos a la información espacial que serán de cumplimiento obligatorio por parte de productores, usuarios y administradores de geoinformación (SENPLADES, 2013, p. 5).

\section{Consideraciones técnicas de la IDE de Ecuador}

Las metas que el Gobierno visualiza, pueden concretarse afirmando que éste persigue:

- Que la geoinformación, esté al alcance de todos los posibles usuarios. Esto es lo que recomiendan las instituciones internacionales (GSDI, GeoSUR, UGGIS, INSPIRE, entre otras). Esta información debe atenerse al Principio de Publicidad de la Información Pública (LOTAIP, 2004, Art. 1) que determina el derecho de las personas a su acceso, considerando información pública toda aquélla que se encuentre en poder de las instituciones del Estado.

- Que se utilice software libre, según el principio de adecuación tecnológica, acordada en la Carta Iberoamericana de Gobierno Electrónico (CLAD, 2007) y conforme con el Decreto Ejecutivo 1014 que establece como política pública para la Administración el uso de Software Libre en sus sistemas informáticos, con el objeto de garantizar que el conocimiento público no sea privatizado y que no exista dependencia de tecnología extranjera.

- Que se cumpla el mandato oficial referido a que cada institución genere la geoinformación que le corresponda y la comparta de manera interoperable con las limitaciones que imponga la ley (SENPLADES, 2013). La definición de Interoperabilidad dada por la Norma de Servicios de Datos Geográficos ISO 19119:2005, establece que es "la capacidad para comunicar, ejecutar programas, o transferir datos entre varias unidades funcionales de forma que se requiera del usuario poco o ningún conocimiento de las características únicas de esas unidades".

- La IDE nacional de Ecuador debe permitir a los usuarios acceder a través de internet a los datos geográficos generados por todas las instituciones oficiales y ofrecer herramientas para gestionarlos. El objetivo es que las decisiones que se puedan tomar sobre un mismo territorio estén avaladas por la oficialidad de la información geográfica publicada por cada institución responsable. En el país, el 
CONAGE, tiene el encargo de poner en marcha la Infraestructura Ecuatoriana de Datos Geoespaciales (IEDG) (Decreto Ejecutivo No. 2250, Art. 2, 2004).

Bajo las anteriores consideraciones, obligatorias para las entidades estatales y tomando como punto de partida el año 2010, fecha en la que se publican las Políticas Nacionales de Información Geoespacial, 18 instituciones del sector público ecuatoriano (Cuadro 1), fueron responsabilizadas de generar los 26 conjuntos de Datos Geográficos Marco definidos para el país. De igual forma, debían estar a disposición de los usuarios de manera interoperable los datos generados, con la premisa del uso de software libre y de forma que no existan limitaciones técnicas para el manejo de la misma. Además, dentro de los Datos Geográficos Marco, se establecieron otros conjuntos de datos denominados de "valor agregado" cuyos responsables no están especificados debido a que pueden ser generados por más de un responsable.

Pero, ¿ha sido posible para las instituciones públicas cumplir con estas políticas o luego de seis años aún no están implementadas a nivel institucional? ¿Cuáles han sido las políticas que se han adaptado a todas las instituciones? y ¿Cuáles son las de más difícil cumplimiento? Además, ¿es importante conocer de forma general los problemas de los usuarios al utilizar la IG objeto de estas políticas?

\section{Objetivos}

El presente estudio pretende evaluar el alcance de las Políticas Nacionales de Información Geoespacial que se orientan hacia la generación y actualización, uso, difusión y mecanismos de entrega, intercambio y venta de la geoinformación (SENPLADES, 2013, p. 12). De igual forma pretende evidenciar el cumplimiento de la política pública sobre el uso de software libre. Para alcanzar el objetivo general se establecen dos objetivos específicos:

- Analizar el porcentaje de las instituciones públicas vinculadas a la generación de Datos Geográficos Marco, que han avanzado en el cumplimiento de parte de las Políticas de Información Geoespacial vinculadas a: generación y actualización, uso, difusión y mecanismos de entrega, intercambio y venta de la geoinformación.

- Evaluar el porcentaje de instituciones que usan software libre en lo referente a sistemas de información geográfica y bases de datos geográficas.

\section{Metodología}

Después de analizar las Políticas Nacionales de Información Geoespacial, se seleccionaron los ítems de cada política general que (a) estuvieran relacionados 
directamente con la gestión de IG y la implementación de las IDE y (b) que fueran parametrizables y medibles mediante la técnica de la encuesta. Con este propósito se diseñó un cuestionario dirigido a las instituciones oficiales generadoras de geoinformación con 26 preguntas, 10 cerradas y 16 semiabiertas (con opción a especificar). El cuestionario fue cumplimentado por 20 de las 29 instituciones responsables de los Datos Geográficos Marco (26 definidas y 3 responsables de datos de valor agregado), establecidas con base en la clasificación del CONAGE, que indica además la competencia de cada institución y la potestad de generación o apoyo a la generación. (Cuadro 1).

La encuesta se realizó a través de internet usando herramientas disponibles en la web 2.0; los ítems seleccionados de cada política y las preguntas relacionadas de la encuesta se detallan a continuación.

\section{Política 1.- Generación y actualización de geoinformación}

- Numeral 1.4. Cada institución productora y/o custodia debe producir, mantener, actualizar y difundir la información geoespacial, de acuerdo con su competencia (preguntas 11, 14, 15 y 22).

- Numeral 1.8. Todas las instituciones productoras y/o custodias de información geoespacial deben generar los metadatos de acuerdo con la normativa vigente, precautelando la propiedad intelectual del titular (pregunta 11).

- Numeral 1.10. Toda información geoespacial debe fundamentarse en los datos geográficos marco (pregunta 13).

- Numeral 1.11. Toda información geoespacial debe estar estructurada de acuerdo con el catálogo de objetos nacional vigente (pregunta 16).

- Numeral 1.12. Las instituciones productoras y/o custodias de información geoespacial deben contar con una base de datos geográfica estructurada, basada en el catálogo de objetos nacional vigente (preguntas 19 y 20).

- Numeral 1.15. Las instituciones públicas custodias de información geoespacial que hayan creado, creen o administren bases de datos deben obligatoriamente integrar su información al Sistema Nacional de Información (SNI) coordinado por la SENPLADES (validada vía web).

\section{Política 2.- Uso de la geoinformación}

- Numeral 2.1. Toda persona natural o jurídica debe utilizar como insumo la información geoespacial oficial (preguntas 13 y 14). 


\section{Política 3.- Difusión de la geoinformación}

- Numeral 3.2. Todas las instituciones custodias de información pública deben garantizar el acceso a su información, salvo que ésta sea declarada secreta, reservada y confidencial (preguntas 13 y 14).

- Numeral 3.4. Toda institución u organización propietaria y/o custodia de información geoespacial debe contar con una Infraestructura de Datos Espaciales (IDE) que garantice el acceso a los servicios de la información que le compete, enlazada a la IEDG, facilitando el acceso, búsqueda, visualización y descarga de la información (preguntas 6 y 7).

\section{Política 4.- Entrega, intercambio y venta de geoinformación}

- Numeral 4.3. Los productores y/o custodios de información geoespacial deben obligatoriamente entregar sus productos, con sus respectivos metadatos (pregunta 11).

Política pública sobre uso de software libre (preguntas 18 y 19).

\section{Cuadro 1}

Datos Geográficos Marco e Instituciones responsables (en negrita, las instituciones que participan en el presente estudio)

\begin{tabular}{llll}
\hline \multicolumn{1}{c}{ Datos } & \multicolumn{1}{c}{$\begin{array}{c}\text { Institución } \\
\text { Competente }\end{array}$} & \multicolumn{1}{c}{$\begin{array}{c}\text { Institución } \\
\text { Generadora }\end{array}$} & $\begin{array}{c}\text { Institución de } \\
\text { Apoyo }\end{array}$ \\
\hline DATOS FUNDAMENTALES & IGM & \\
\hline $\begin{array}{l}\text { Referencia Geodésica } \\
\text { Límite internacional }\end{array}$ & MMRREE & MMRREE, IGM & \\
$\begin{array}{l}\text { Límite marítimo } \\
\text { internacional }\end{array}$ & MMRREE & MMRREE, INOCAR & \\
Límite provincial & CONALI & CONALI & INEC, IGM \\
Límite cantonal & CONALI & CONALI & INEC, IGM \\
Límite parroquial & CONALI & CONALI, Municipios & INEC, IGM \\
Altimetría & IGM & IGM & \\
Batimetría & INOCAR & INOCAR & \\
Datos de sensores remotos & IEE, IGM, FAE & IEE, IGM, FAE & \\
Infraestructura vial & MTOP, Consejos & MTOP, Consejos & IGM \\
& Provinciales & Provinciales & \\
Redes hidrográficas & IGM, INOCAR & IGM, INOCAR & \\
& & IGM, INEC, Ministerio & \\
Nombres geográficos & IGM & Coordinador de & \\
& & Patrimonio y Cultura & \\
\hline & & & \\
\hline & & &
\end{tabular}


DATOS BÁSICOS

\begin{tabular}{|c|c|c|c|}
\hline Geoestadísticos & INEC & INEC & $\begin{array}{l}\text { Ministerios } \\
\text { competentes }\end{array}$ \\
\hline \multicolumn{4}{|l|}{ Recursos Naturales } \\
\hline $\begin{array}{l}\text { Geologría, geomorfología, } \\
\text { recursos minerales }\end{array}$ & INIGEMM & \multicolumn{2}{|l|}{ INIGEMM, IEE } \\
\hline Hidrología & $\begin{array}{l}\text { SENAGUA, } \\
\text { INAMHI, } \\
\text { INOCAR }\end{array}$ & \multicolumn{2}{|l|}{$\begin{array}{l}\text { SENAGUA, } \\
\text { INAMHI, INOCAR }\end{array}$} \\
\hline Meteorología & INAMHI & \multicolumn{2}{|l|}{ INAMHI, INOCAR, DAC } \\
\hline Hidrogeología & $\begin{array}{l}\text { SENAGUA, } \\
\text { INAMHI, } \\
\text { INIGEMM }\end{array}$ & \multicolumn{2}{|l|}{$\begin{array}{l}\text { SENAGUA, } \\
\text { INAMHI, } \\
\text { INIGEMM, IEE }\end{array}$} \\
\hline Edafología & MAGAP & \multicolumn{2}{|l|}{ MAGAP, IEE } \\
\hline Uso de la tierra & MAGAP & \multicolumn{2}{|l|}{ MAGAP, IEE } \\
\hline Cobertura vegetal & MAE & \multicolumn{2}{|l|}{ MAE, IEE } \\
\hline Cuenca hidrográfica & SENAGUA & \multicolumn{2}{|l|}{ SENAGUA } \\
\hline $\begin{array}{l}\text { Sistema Nacional de Áreas } \\
\text { Protegidas }\end{array}$ & MAE & \multicolumn{2}{|l|}{ MAE } \\
\hline Biodiversidad & MAE & MAE & $\begin{array}{l}\text { Museo de } \\
\text { Ciencias } \\
\text { Naturales } \\
\end{array}$ \\
\hline \multicolumn{4}{|l|}{ Catastro } \\
\hline Catastro Urbano & $\begin{array}{l}\text { MIDUVI, } \\
\text { Municipios }\end{array}$ & Municipios & AME \\
\hline Catastro rural & $\begin{array}{l}\text { Municipios, Juntas } \\
\text { parroquiales }\end{array}$ & $\begin{array}{l}\text { Municipios, Juntas } \\
\text { parroquiales }\end{array}$ & $\begin{array}{l}\text { MAGAP, } \\
\text { SIGTIERRAS }\end{array}$ \\
\hline Amenazas & SNGR & $\begin{array}{l}\text { IGM, INOCAR, } \\
\text { INIGEMM }\end{array}$ & \\
\hline \multicolumn{4}{|c|}{ DATOS DE VALOR AGREGADO (no se establecen instituciones responsables) } \\
\hline \multicolumn{4}{|l|}{ Educación } \\
\hline \multicolumn{4}{|l|}{ Salud } \\
\hline \multicolumn{4}{|l|}{ Cultura } \\
\hline \multicolumn{4}{|l|}{ Turismo } \\
\hline \multicolumn{4}{|l|}{ Vivienda } \\
\hline \multicolumn{4}{|l|}{ Espacio aéreo y marino } \\
\hline Entre otros... & & & \\
\hline
\end{tabular}

Fuente: Adaptación de Datos Geográficos Marco (CONAGE, 2013).

Considerar que CELIR ahora es CONALI y CLIRSEN actualmente es IEE.

Para el estudio se consideró en datos de valor agregado al Ministerio de Salud, Dirección de Aviación Civil y Comando Conjunto de Fuerzas Armadas. 


\section{Resultados y discusión}

\section{Generación y actualización de geoinformación}

Política 1 numeral 1.4. Se encuentran involucrados tres aspectos:

- Producción y mantenimiento de información geoespacial. De los resultados de la encuesta se determinó que el 100\% de los Datos Geográficos Marco son producidos y mantenidos por la institución competente y/o generadora, según lo definido en el marco legal de cada institución.

- Actualización de la información. La Figura 1 muestra los principales problemas identificados por las instituciones usuarias de la información geográfica. El 75\% (15 de 20) de las instituciones considera que el mayor inconveniente es la falta de actualización de la IG.

\section{En general ¿Qué problemas ha observado con el uso de la Información Geográfica?}

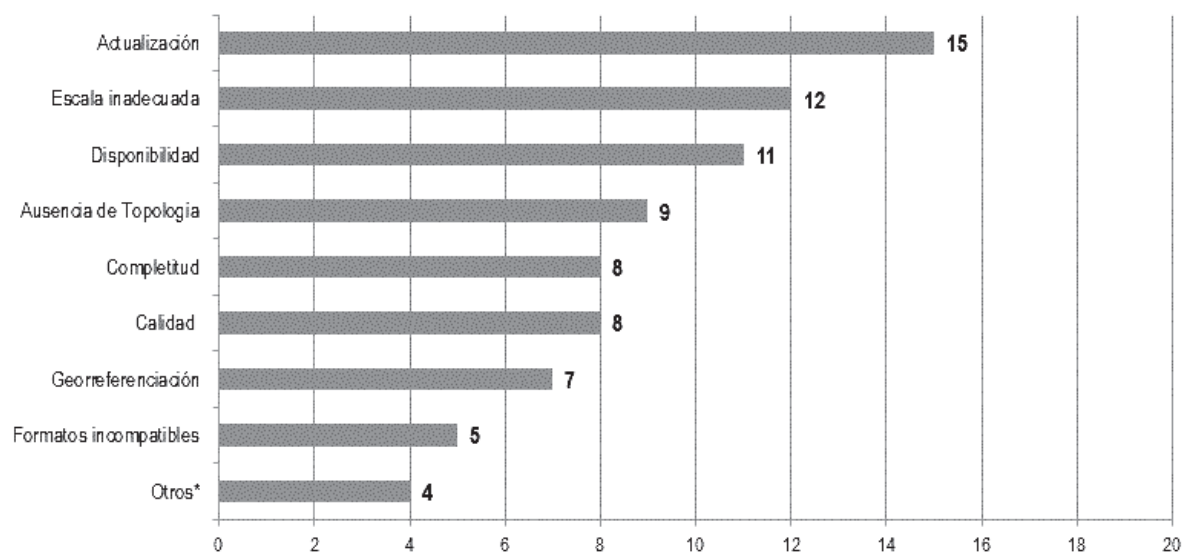

Figura 1. Problemas observados por los usuarios con la información geográfica.

- Divulgación de la información; en promedio, por cada dato considerado en el estudio, existen 11 instituciones (que requieren el dato) que se enteraron de su existencia a través de una publicación, reunión o contacto con algún funcionario de la institución generadora, esto es el 55\% de las instituciones. El 15\% (3 de 20) lo hizo por medio del geoportal institucional y un $10 \%$ ( 2 de 20$)$ por una visita a la institución. El restante corresponde a otros medios no especificados. $20 \%$ 


\section{¿Porqué medio se enteró de la existencia de los datos?}

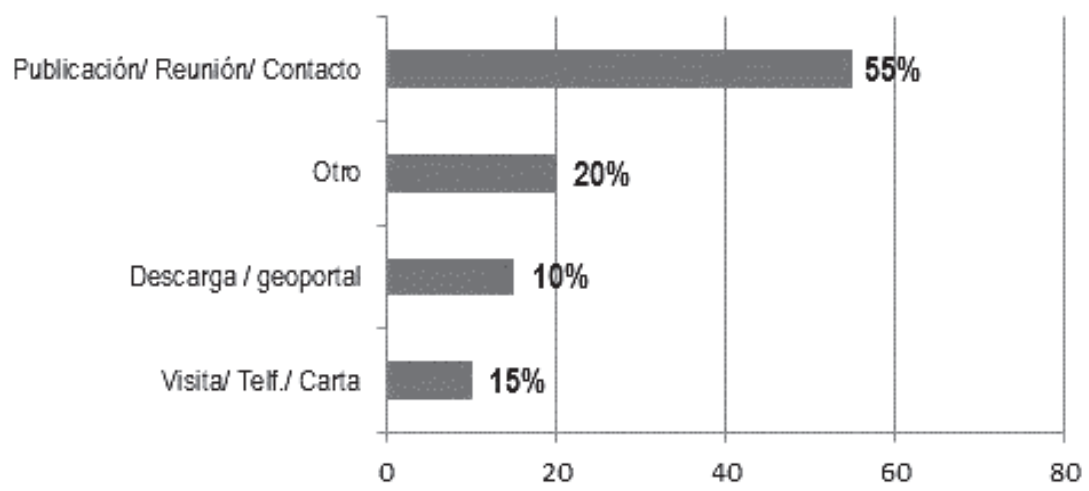

Figura 2. Medios utilizados para conocer de la existencia de información geográfica.

Este resultado concuerda con lo indicado en "Geoinformación institucional en el Ecuador: acceso y uso" (León-Pazmiño et al., 2016), donde se establece que el medio más utilizado para compartir la información entre instituciones públicas es a través de disco externo/CD con el 51\%, lo que implica necesariamente una o más visitas a la institución generadora con el consiguiente gasto de tiempo. Solo el $25 \%$ lo hace por medio de descarga/geoportal de forma directa, debido a un conocimiento previo de la existencia de la información.

\section{Generación de geoinformación con metadatos PEM por institución}

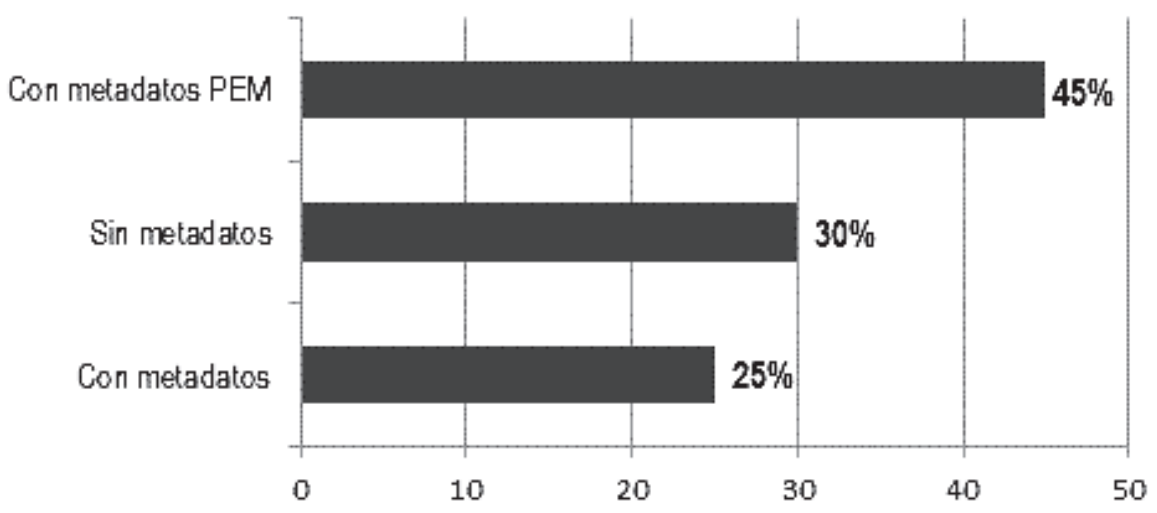

Figura 3. Instituciones que generan datos con sus respectivos metadatos. 
Política 1 numeral 1.8. En el tema de generación de información con metadatos, se estableció que, de los 34 conjuntos de datos geográficos marco consultados (sin considerar los de educación y turismo debido a que las instituciones oficiales no participaron del presente estudio), el $62 \%$ son generados por la institución competente mediante el Perfil Ecuatoriano de Metadatos (PEM), 12\% con metadatos con diferente normativa y $26 \%$ sin metadatos. A nivel institucional, $45 \%$ de las instituciones (9 de 20) indicaron que generan su información con PEM, 25\% (5 de 20) con metadatos no PEM y 30\% (6 de 20) sin metadatos (Figura 3).

Política 1 numeral 1.10. De las respuestas obtenidas, se evidencia que el 100\% de las instituciones públicas participantes, utilizan los Datos Geográficos Marco para generar su geoinformación.

Política 1 numeral 1.11. El 65\% (13 de 20) instituciones usan el catálogo de objetos nacional para la generación de su información, el 10\% (2 de 20) usan un catálogo no conforme con el catálogo nacional y el 25\% (5 de 20) no usa catálogo.

Política 1 numeral 1.12. El 90\% (18 de 20) de las instituciones generan bases de datos geográficas, pero solo 13 de las 18 usan el Catálogo de Objetos Nacional, por tanto el 65\% (13 de 20) cumplen la política.

Política 1 numeral 1.15. La evaluación de este numeral se realizó vía web, a través de la verificación de integración de la información generada por las instituciones al Sistema Nacional de Información (SNI), con corte al 17 de agosto de 2016. Se encontró que existen tres opciones de acceso a la IG en la página web del SNI: (a) por medio del integrador geográfico <http://sni.gob.ec/ integrador_geografico/>, desde donde se puede acceder a los geoportales y visores institucionales; (b) desde la descarga de archivos de información geográfica, que corresponde a un repositorio de datos geográficos <http://sni.gob.ec/coberturas $>$ y (c) desde el catálogo de datos abiertos geográficos < http://sni.gob.ec/metadatos $>$. En el primer caso, se encontró que sólo el 20\% (4 de 20) de las instituciones participantes tienen el link habilitado para acceder a su geoportal y/o visor, 15\% (3 de 20) tienen un link erróneo y el resto no lo tiene indexado. En el caso de acceso por descarga de archivos, el 50\% de las instituciones ponen a disposición su información de forma total o parcial. Finalmente el 10\% (2 de 20) permiten acceder a su información a través de las herramientas de un catálogo de objetos.

\section{Uso de geoinformación}

Politica 2 numeral 2.1. Se considera igual a la Política1 numeral 1.10, es decir el $100 \%$ de las instituciones usan la información oficial. 


\section{Difusión de geoinformación}

Política 3 numeral 3.2. En León-Pazmiño et al., quedó evidenciado que el 100\% de las instituciones comparten sus datos, con un índice de compartición del 64\% (2016). Sin embargo, al ser el objetivo de las políticas fomentar la implementación de las IDE, es importante considerar la forma en que la información se pone a disposición en cada institución, pues en la Política 1 numeral 1.4, se definió que el $25 \%$ de las instituciones brinda acceso a través de descarga directa del geoportal. Debe considerarse también la posibilidad de obtener la IG por medio del SNI.

Política 3 numeral 3.4. No existe una definición generalizada de los elementos que conforman una IDE, aunque la mayoría de autores coinciden en que son: datos, hardware y software, metadatos, tecnologías, estándares, acuerdos entre productores, personal, organización, marco legal/institucional, políticas y usuarios (Abad et al., 2012) (Iniesto y Núñez, 2014). Para la presente investigación se consideró que si la Institución disponía al menos de un Geoportal en funcionamiento conforme con estándares ISO y especificaciones OGC, "cumplía" con los elementos de una IDE. El resultado establece que el $45 \%$ de las instituciones participantes en el estudio cuentan con una IDE (León-Pazmiño et al., 2016).

\section{Entrega, intercambio y venta de geoinformación}

Política 4 numeral 4.3. De la Figura 3 se puede afirmar que el 70\% (14 de 20) de las instituciones entrega su información con metadatos, pero solo el 45\% (9 de 20) con la normativa ecuatoriana vigente.

Sobre la política de uso de software libre, en lo relacionado a sistemas de información geográfica, los resultados determinaron que el 95\% de las instituciones (19 de 20) utilizan el software comercial ArcGis (Figura 4). Adicionalmente las instituciones usan además otro software, que puede ser libre o comercial. QGIS, es el software libre más utilizado con $65 \%$ de instituciones, seguido de gvSIG, con el $25 \%$.

Respecto de las bases de datos espaciales, el software PostGRES/PostGIS, que es software libre, es el más utilizado, con el $65 \%$ de las instituciones (13 de 20) (Figura 5). De estas, 5 utilizan además otro software comercial como ORACLE y MySQL Spatial. El restante 35\% (7 de 20) indicó que no usan bases de datos espaciales. 


\section{Sistemas de Información Geográfica Utilizados por Institución}

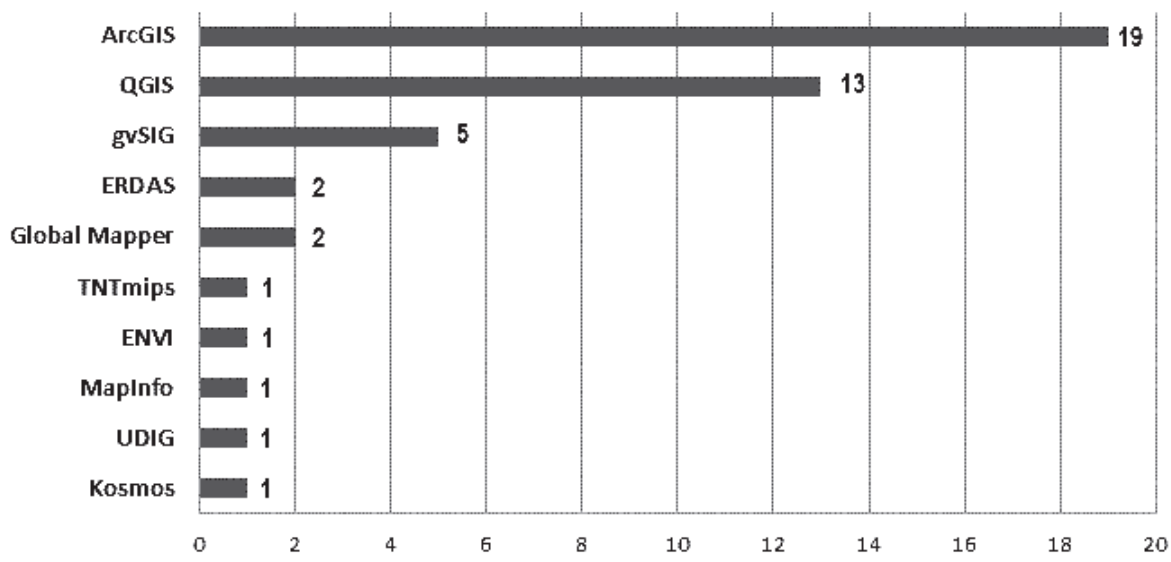

Figura 4. Software de Sistemas de Información Geográfica utilizados.

\section{Bases de Datos más Utilizado por Institución}

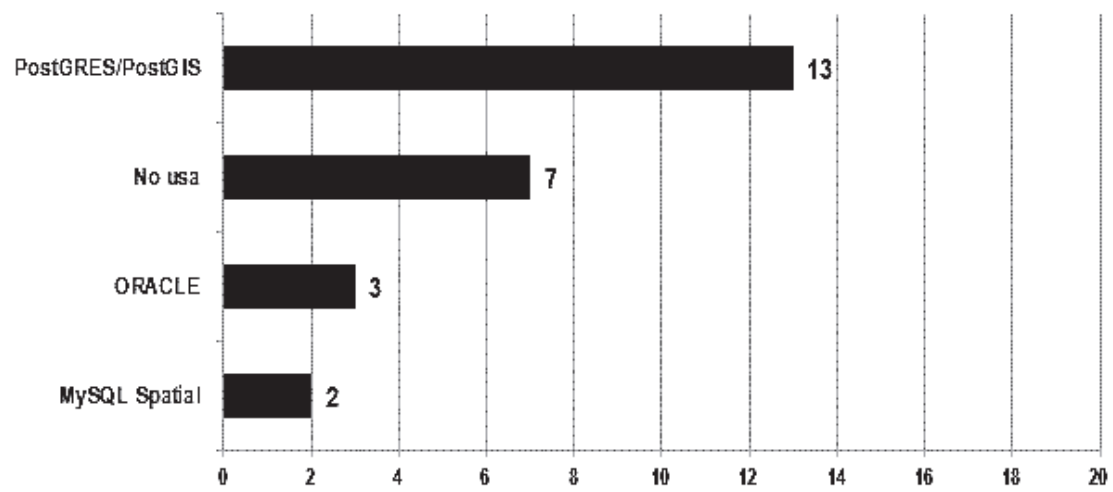

Figura 5. Uso de software de Base de Datos Geográfica.

A fin de consolidar los resultados de la evaluación, se elaboró una matriz de valoración con cada política, con base en los datos de la encuesta, cuyos resultados se presentan en la Figura 6.

En resumen, si se realiza el promedio por Institución, el porcentaje de cumplimiento de las políticas consideradas en el estudio, se obtiene que: cinco 
instituciones tienen un porcentaje superior al 80\%, 11 se encuentran en el rango que va del $80 \%$ al $50 \%$ y 4 obtuvieron un porcentaje menor al $50 \%$. Con estos rangos se realizó una clasificación que comprende las clases de: cumple, cumple parcialmente y no cumple (Figura 7).

\section{Cumplimiento de Políticas Nacionales de Geoinformación Evaluadas}

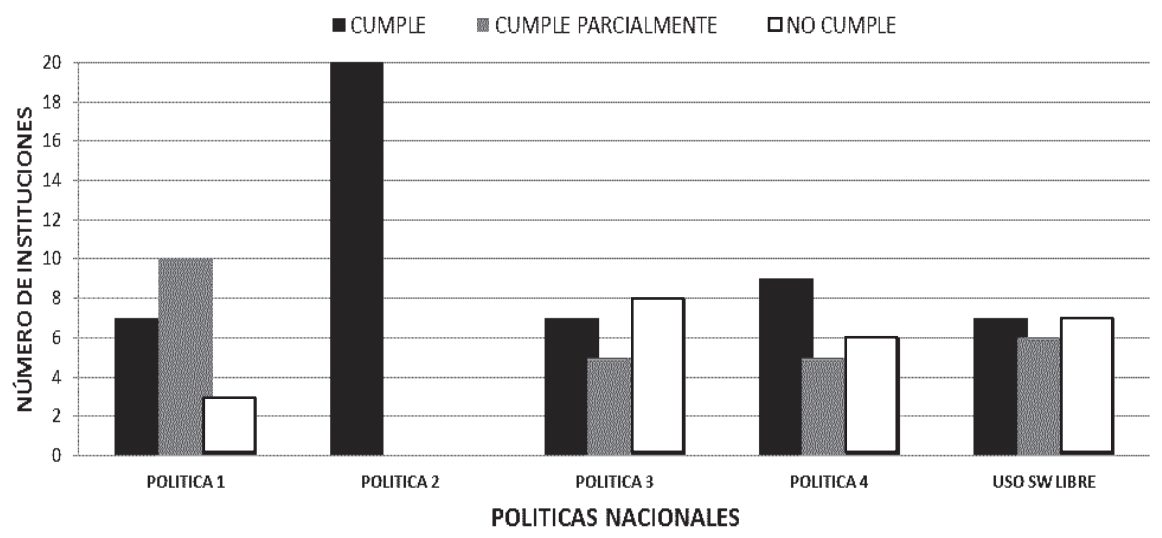

Figura 6. Número de instituciones según el cumplimiento de las Políticas Nacionales de Información Geoespacial evaluadas.

\section{Porcentaje de Cumplimiento por Institución}

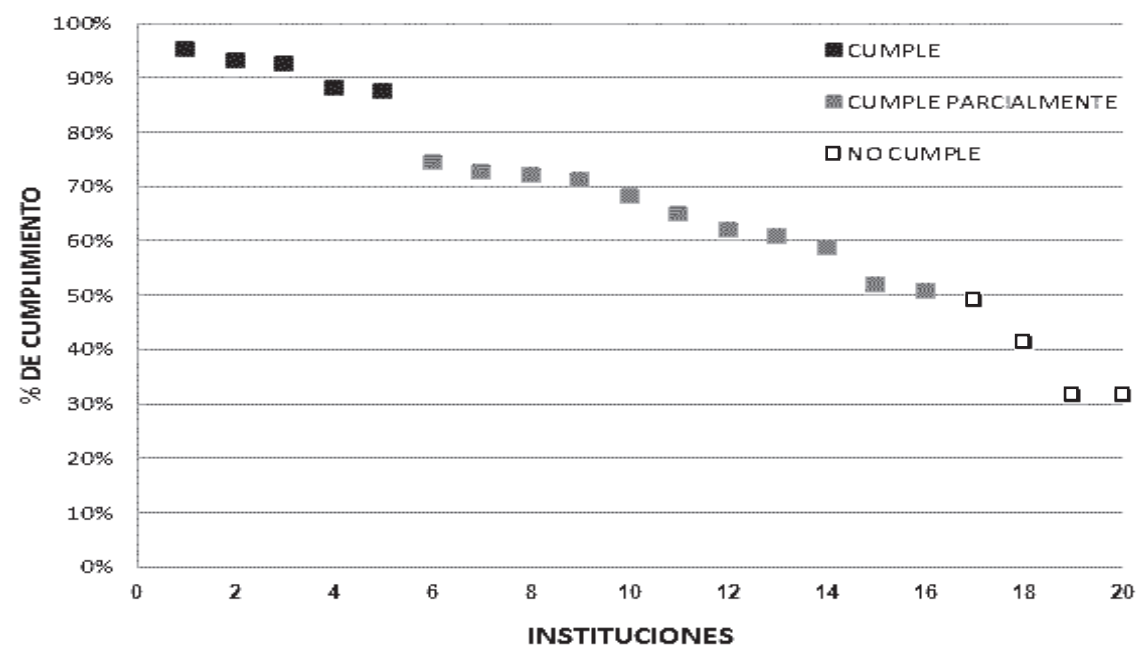

Figura 7. Porcentaje de cumplimiento de las Políticas evaluadas por Institución. 


\section{Conclusiones}

En lo referente a la producción y mantenimiento de la información, se concluye que todas las instituciones participantes cumplen con la generación y mantenimiento de la información que les corresponde según su responsabilidad. No así con la actualización, puesto que el $75 \%$ de las instituciones considera que existe falta de actualización de la información geoespacial. La difusión de la información, evidenciada por un $13 \%$ de instituciones que conocieron la existencia de la información a través del geoportal y un $25 \%$ que accedió a sus datos a través del mismo medio, refleja que aún no existe un buen nivel de conocimiento por parte del usuario de la disponibilidad de servicios geográficos web.

La generación de información geoespacial con metadatos PEM, alcanza al 62\% de los datos analizados, con el $45 \%$ de las instituciones, lo que permite afirmar que cerca del 50\% de las instituciones han adoptado el Perfil Ecuatoriano de Metadatos.

La política 1 numeral 1.15 que trata sobre la integración de la información en el SNI, tiene el nivel más bajo de cumplimiento de las políticas analizadas, porque apenas 4 de 9 instituciones que cuentan con geoportal conforme con ISO y OGC, tienen indexado un link en el SNI para acceder a su geoportal y solo el $50 \%$ de las instituciones participantes en el estudio (10 de 20) tienen disponible su información para descarga a través del SNI.

El 100\% de las instituciones afirma utilizar los datos geográficos marco como base para la generación de su geoinformación, por lo tanto existe un cumplimiento total de la política 2.1 .

En la evaluación de la política 3, numeral 3.2 (acceso a la información pública), se consideró el medio a través del cual se pone a disposición la información, siendo los más relevantes los realizados por medios web, pero solo siete instituciones lo han implementado por medio de geoportal, integración al SNI o aplicativos web, el resto lo cumple pero por medios físicos.

La política que fomenta el uso del catálogo de objetos nacional vigente se vincula con la política de generación de bases de datos geográficas. Las dos presentan un buen nivel de cumplimiento con el $65 \%$ de las instituciones.

$\mathrm{Al}$ comprobar que el $45 \%$ de las instituciones cuentan con un geoportal que cumple la normativa ISO y OGC, se evidencia que hay un buen número de instituciones que cumplen la política 3, numeral 3.4. Sin embargo durante la realización de la entrevista para el llenado de la encuesta, al menos dos instituciones indicaron que contaban con un geoportal en funcionamiento, pero debido a cambios de personal y de tecnología, éstos dejaron de funcionar, es decir que probablemente existe una disminución en la cantidad de instituciones que cuentan con geoportal, en comparación con años anteriores, pero al no existir datos, no es posible comprobarlo. 
Con respecto a la política de uso de software libre, en el tema de bases de datos geográficas, el nivel de cumplimiento es alto, dado que 13 de 20 instituciones, que afirmaron usarlas, optan por una de software libre. No así, en el caso de los sistemas de información geográficos, donde el $95 \%$ de las instituciones (19 de 20) opta por un software comercial. Sin embargo se debe resaltar que 14 de 19, utiliza como software alternativo o complementario un software libre.

El 25\% (5 de 20) de las instituciones encuestadas tiene un cumplimiento de las políticas evaluadas superior al $80 \%$ y $55 \%$ (11 de 20) instituciones tienen un cumplimiento superior al 50\%, en un periodo de 6 años aproximadamente (20102016).

En resumen, el promedio general de cumplimiento de las Políticas Nacionales de Información Geoespacial consideradas en el estudio y de uso de software libre, es del $66 \%$. Las políticas de difusión e intercambio de geoinformación son las de menor cumplimiento a pesar de que según la definición de IDE nacional, son de gran importancia para la implementación de la Infraestructura Ecuatoriana de Datos Geoespaciales (IEDG).

\section{Agradecimientos}

El presente trabajo fue realizado en el marco del programa PROMETEO en el que el Instituto Geográfico Militar (IGM), Ecuador participa como institución de acogida, demostrando que ha apostado por la investigación en temas geográficos, a favor de la transferencia de conocimiento y tecnología; siendo el principal objeto de su misión.

Nuestra sincera gratitud al programa PROMETEO, al IGM, a quienes nos apoyaron con sus comentarios y a los que nos colaboraron con su tiempo en el llenado de la encuesta, insumo básico del análisis realizado.

\section{Bibliografía}

Abad Power, P.; Bernabé, M. y Rodríguez Pascual, A. (2012). "Capítulo 2. Compartir: la solución está en las Infraestructuras de Datos Espaciales", en Bernabé, M. y López, C., Fundamentos de las Infraestructuras de Datos Espaciales, Serie Científica, UPM Press, Madrid, España, pp. 41-53.

Béjar, R.; Latre, M.A.; Nogueras-Iso, J.; Muro-Medrano, P. y Zarazaga-Soria, J. (2009). "Systems of Systems as a Conceptual Framework for Spatial Data Infrastructures", International Journal of SDI Research. 4:201-217 [en línea], revisado en $<$ http://ijsdir.jrc.ec.europa.eu/index.php/ijsdir/article/view/124>

Bernabé-Poveda, M.A. y López-Vázquez, C.M. (2012). "Prefacio", en Fundamentos de las Infraestructuras de Datos Espaciales, Serie Científica, UPM Press, Madrid, España, pp. 17-27. 
CLAD, Centro Latinoamericano de Administración para el Desarrollo (2007). "Carta Iberoamericana de Gobierno Electrónico”, Resolución No. 18, 10 de noviembre de 2007, Santiago, Chile, aprobada por la XVII Cumbre Iberoamericana de Jefes de Estado y de Gobierno, [en línea], revisado en: <http://old.clad. org/documentos/declaraciones/cartagobelec.pdf>

CONAGE, Consejo Nacional de Geoinformática (2013). "Políticas Nacionales de Información Geoespacial", Registro Oficial, No. 269, del 01 de septiembre del 2010 reformadas con Resolución No. 003-CONAGE-2013, 24 de julio de 2013, Quito, Ecuador.

Decreto Ejecutivo No. 2250 (2004). Decreto Ejecutivo de creación del Consejo Nacional de Geoinformática, de 11 de noviembre del 2004. Publicado en el Registro Oficial No. 466 de 22 de noviembre del 2004, Quito, Ecuador.

IGN, Instituto Geográfico Nacional (2012). Curso e-learning de Infraestructuras de Datos Espaciales. Unidad 1: Componentes de una IDE y marcos legales. Madrid-España, [en línea], revisado en: <https://www.ign.es/ign/resources/ cartografiaEnsenanza/ideeEso/I-IDE/I-IDE/ini_op5.html>

Iniesto, M. y Nuñez, A. (2014). "Introducción a la Infraestructura de Datos Espaciales", M. Fomento, Madrid, España.

León-Pazmiño, M.F.; Narváez-Benalcázar, R.; Bernabé-Poveda, M.A. y González Campos, M.E. (2016). “Geoinformación Institucional en el Ecuador: acceso y uso”, Revista Geoespacial, ESPE, 13(1), Quito, Ecuador, pp. 40-58.

LOTAIP, Ley Orgánica de Transparencia y Acceso a la Información Pública (2004). Ley 24, Registro Oficial Suplemento 337 de 18 de mayo del 2004, Quito, Ecuador.

Manrique, M.T. y Manso, M.A. (2012). "Los Geoportales. Perspectiva desde la usabilidad", Fundamentos de las Infraestructuras de Datos Espaciales, UPM Press, Serie Científica, pp. 393-403.

OGC, Open Geospatial Consortium (2003). "OpenGIS Reference Model." pp4. Reference: OGC 03-040. Version 0.1.3. Kurt Buehler (ed). Version Web (2.1). [en línea], revisado en <http://www.opengeospatial.org/standards/orm>

SENPlADES, Secretaría Nacional de Planificación y Desarrollo. (2009). "Plan Nacional para el Buen Vivir 2009-2013”, Quito, Ecuador [en línea], revisado en $<$ https://issuu.com/buen-vivir/docs/pnbv_2009-2013>

SENPLADES, Secretaría Nacional de Planificación y Desarrollo (2013). Estándares de información geográfica, tomo I, Quito, Ecuador, pp. 1-50 [en línea], revisado en $<$ http://sni.gob.ec/documentos-conage> 